\section{ORIGINAL RESEARCH}

\author{
Y. Ge \\ J.H. Jensen \\ H. Lu \\ J.A. Helpern \\ L. Miles \\ M. Inglese \\ J.S. Babb \\ J. Herbert \\ R.I. Grossman
}

\title{
Quantitative Assessment of Iron Accumulation in the Deep Gray Matter of Multiple Sclerosis by Magnetic Field Correlation Imaging
}

\begin{abstract}
BACKGROUND AND PURPOSE: Deposition of iron has been recognized recently as an important factor of pathophysiologic change including neurodegenerative processes in multiple sclerosis (MS). We propose that there is an excess accumulation of iron in the deep gray matter in patients with MS that can be measured with a newly developed quantitative MR technique-magnetic field correlation (MFC) imaging.

MATERIALS AND METHODS: With a 3T MR system, we studied 17 patients with relapsing-remitting MS and 14 age-matched healthy control subjects. We acquired MFC imaging using an asymmetric single-shot echo-planar imaging sequence. Regions of interest were selected in both deep gray matter and white matter regions, and the mean MFC values were compared between patients and controls. We also correlated the MFC data with lesion load and neuropsychologic tests in the patients.
\end{abstract}

\begin{abstract}
RESULTS: MFC measured in the deep gray matter in patients with MS was significantly higher than that in the healthy controls $(P \leq .03)$, with an average increase of $24 \%$ in the globus pallidus, $39.5 \%$ in the putamen, and $30.6 \%$ in the thalamus. The increased iron deposition measured with MFC in the deep gray matter in the patients correlated positively with the total number of MS lesions (thalamus: $r=0.61, P=.01$; globus pallidus: $r=0.52, P=.02$ ). A moderate but significant correlation between the MFC value in the deep gray matter and the neuropsychologic tests was also found.
\end{abstract}

CONCLUSION: Quantitative measurements of iron content with MFC demonstrate increased accumulation of iron in the deep gray matter in patients with MS, which may be associated with the disrupted iron outflow pathway by lesions. Such abnormal accumulation of iron may contribute to neuropsychologic impairment and have implications for neurodegenerative processes in MS.

A lthough most lesions associated with demyelinating changes in multiple sclerosis (MS) are seen in white matter, recent postmortem and in vivo studies have shown increased evidence that gray matter is also significantly involved. ${ }^{1,2}$ Neurodegenerative changes have been identified in both histopathologic and advanced imaging studies in patients with MS, in which substantial neuronal loss is indicated by decreased neuronal attenuation, ${ }^{3} \quad N$-acetylaspartate (NAA), ${ }^{4,5}$ and volume in both cortical gray matter and deep gray matter nuclei, ${ }^{6,7}$ which suggests a significant neuronal pathologic process in patients with MS. Deep gray matter structures such as the thalamus, putamen, globus pallidus, and caudate play significant roles in processing motor, sensory, cognitive, and emotional information. Therefore, injury of these structures, including neuropsychologic impairment, may significantly contribute to the disease. However, little is known about the causes and pathophysiology of neurodegenerative changes in the deep gray matter of MS.

Previous studies have shown increased iron in the nuclei of

Received December 18, 2006; accepted after revision March 1, 2007

From the Center for Biomedical Imaging (Y.G., J.H.J., H.L., J.A.H., L.M., M.I., J.S.B., R.I.G.), Department of Radiology, New York University Medical Center, New York, NY; and the Department of Neurology (J.H.), MS Care Center, Hospital for Joint Diseases, New York University School of Medicine, New York, NY.

This paper won the 2007 Cornelius G. Dyke Memorial Award and was presented the Annual Meeting of the American Society of Neuroradiology, June 11, 2007, Chicago, III.

This work was supported by grants R37 NS29029-11 and R21/R33 EB003305-01 from the National Institutes of Health and NCRR M01 RR00096 (GCRC).

Please address correspondence to Yulin Ge, MD, Department of Radiology/Center for Biomedical Imaging, New York University School of Medicine, 650 First Ave, 6th Floor, Room 615, New York, NY 10016; e-mail: yulin.ge@med.nyu.edu

DOI 10.3174/ajnr.A0646 the basal ganglia, which is thought to be a cause of the neurodegenerative pathologic process in many age-related degenerative disorders such as Parkinson and Alzheimer disease. ${ }^{8}$ Our current understanding of accumulation of iron in these neurodegenerative diseases is incomplete. However, the toxic effects of excess iron in the brain on lipids, carbohydrates, proteins, and nucleic acids have been well established. ${ }^{9}$ In general, gross pathologic examinations and neuroimaging have indicated neuronal loss that is responsible for the regional atrophy in these diseases. In MS, recent research has explored the role of iron in the pathogenesis and neuronal damage by ironinduced oxidative damage. ${ }^{10}$ The iron-catalyzed reaction that leads to damage by free radicals has been reported in studies of the pathogenesis of experimental allergic encephalomyelitis (EAE), ${ }^{11-14}$ an animal model of MS, in which an abnormal histopathologic iron staining was often observed in tissues from EAE models.

In studies on humans, conventional T2-weighted imaging also revealed abnormal hypointense signals in lesions, the basal ganglia, thalamus, and cerebral cortex, which indicates pathologic deposition of iron possibly leading to tissue damage in MS. ${ }^{15,16}$ Neurodegeneration of the deep gray matter may correspond to the cognitive impairment commonly observed in patients with MS. ${ }^{17}$ These findings are important in suggesting that an abnormal deposition of iron plays a significant role in the pathophysiologic process of tissue injury in MS. Previous data analysis for iron assessment on the basis of qualitative rating judged with relative evaluation of signal intensity of structures on T2-weighted imaging may not be appropriate because of the inherent variations of signal intensity in acquisition-to-acquisition MR images. ${ }^{18}$ Bakshi et al $^{15,19}$ 
have quantified the effects of iron deposition on the basis of decreased T2 signal intensities in MS. However, T2-weighted images, especially fast spin-echo sequences, have lower sensitivity and specificity for the evaluation of iron.

We conducted this study to apply a newly developed quantitative MR technique with a unique iron contrast mechanism on the basis of magnetic field correlation (MFC), ${ }^{20-23}$ to assess iron deposition in the brain in patients with MS. MFC has a direct relationship to the microscopic field inhomogeneity (MFI) or field shifts, such as those generated by iron-rich cells with length scales ranging roughly from 1 to $100 \mu \mathrm{m}$. Unlike $\mathrm{R} 2$ and $\mathrm{R} 2^{*}$ techniques that can be influenced by other relaxation mechanisms (eg, dipolar), MFC depends only on MFI and water diffusion. In this study, we investigated whether MFC can quantify abnormal iron deposition that is not apparent on conventional MR imaging in the deep gray matter of patients with MS compared with age-matched healthy control subjects. We also investigated the relationship between the iron level in the deep gray matter and total lesion load as well as neuropsychologic tests in these patients.

\section{Materials and Methods}

\section{Subjects}

We recruited 17 patients (14 women and 3 men) with clinically definite relapsing-remitting MS from consecutive admissions to the MS clinical center in our hospital. The mean age was 44 years (range, $30-57$ years), and the mean duration of the disease at the time MR imaging was performed was 5.5 years (range, 1.3-13.8 years). Two patients have not received any immunosuppressive or immunomodulatory drugs, and 15 were receiving immunomodulating therapy (11 patients on interferon- $\beta 1$ a [Avonex; Biogen, Cambridge, Mass] and 4 on Copolymer-1 [Copaxone; Teva, Petach Tikya, Israel]). Fourteen healthy volunteers ( 9 women, 5 men; mean age, 39 years; range, 23-55 years) served as control subjects and had no previous history of neurologic diseases and no vascular or other abnormalities seen on T1-weighted and T2-weighted MR images.

For neuropsychologic tests, we conducted a traditional consensus battery that yields measures of executive functions, attention, concentration, verbal ability, psychomotor ability, and memory. Sensitive to regional brain dysfunction, this battery is suited for repeated testing and consists of 8 neuropsychologic tests: Wechsler Adult Intelligence Scale (WAIS)-III Digit Symbol-Coding (DS), Rey-Osterrieth Complex Figure Test (ROCFT), Delis-Kaplan Executive Function System (D-KEFS) Verbal Fluency Test (VF), California Verbal Learning Test II (CVLT), WAIS-III Digit Span Backward (DSB), Symbol Digit Modalities Test (SDMT), D-KEFS Color Word Interference Test (CWI), and the Paced Auditory Serial Attention Test (PASAT). We conducted all study protocols within the guidelines from the Institutional Review Board. We also obtained consent from each participant.

\section{MR Imaging and Image Processing}

We performed MR imaging examinations on a clinical 3T Trio unit (Siemens, Malvern, Pa). We acquired the images using our standard protocol for MS study, which includes dual-echo fast spin-echo proton attenuation and T2-weighted imaging (TR/TE1/TE2, 5500/12/99 ms; matrix, $256 \times 256$; pixel size, $0.86 \times 0.86 \mathrm{~mm}^{2}$ ) as well as contrast-enhanced T1-weighted imaging (TR/TE, 660/14 ms). These routine sequences were acquired with 3 -mm-thick contiguous axial sections for lesion identification. We acquired MFC images by per- forming a single-shot echo-planar imaging (EPI) asymmetric echo (ASE) sequence. A square field of view (FOV) of $256 \times 256 \mathrm{~mm}^{2}$ with an acquisition matrix of $128 \times 128 \mathrm{~mm}^{2}$ and a section thickness of 2 $\mathrm{mm}$ was used, which resulted in isotropic voxels. A total of 40 sections were obtained. The bandwidth was $1345 \mathrm{~Hz} /$ pixel with a TR of 2800 $\mathrm{ms}$ and TE of $59 \mathrm{~ms}$. Images were acquired with refocusing pulse time shifts of $\mathrm{t}_{\mathrm{s}}=0,-4,-8,-12$, and $-16 \mathrm{~ms}$, where the negative sign indicates a reduction of the interval between the initial $90^{\circ}$ excitation pulse and $180^{\circ}$ refocusing pulse from the usual spin-echo value of $\mathrm{TE} / 2$. For each set of imaging parameters, 20 images were acquired to increase the signal-to-noise ratio. The scanning time for MFC was 3 minutes and 35 seconds. The sections were oriented axially with a slight tilt to avoid the sinus cavity, a potential source of macroscopic MFI.

The MR images were transferred to a PC-based computer for postprocessing. We performed 3D motion correction using SPM software (Wellcome Department of Imaging Neuroscience, University College London, London, UK). The 20 repetitions were then averaged for each refocusing shift.

The MFC is formally defined by

$$
\operatorname{MFC}(t)=\gamma^{2}\langle B(t)(B)(0)\rangle
$$

where $B(t)$ is the difference between the magnetic field experienced by a water molecule at time $t$ and the uniform background field, $\gamma$ is the proton gyromagnetic ratio, and the angle brackets indicate an averaging over all the water protons within a voxel. The MFC at $t=0$ is simply proportional to the variance for the MFIs. As $t$ increases, the MFC typically decreases because water diffusion tends to reduce the correlation of a water molecule's local magnetic field at different times.

MFC imaging requires asymmetric spin-echo images to be obtained for a fixed acquisition time (ie, TE) but with a range of asymmetry shifts for the $180^{\circ}$ refocusing pulse. The MFC is then determined by fitting the signal intensity $S$ to the formula

$$
S\left(t_{s}\right)=S(0) \exp \left[-2 t_{s}^{2} \cdot \mathrm{MFC}\right],
$$

where TE is the echo time and $t_{s}$ is the refocusing pulse shift. Initial data indicate that the MFC in the brain correlates strongly with the concentration of iron. ${ }^{22,24}$ We generated the MFC maps from the images using in-house MATLAB scripts. Three regions of interest (ROIs) in the deep gray matter-the thalamus, putamen, and globus pallidus - and 3 ROIs in the white matter-the frontal white matter, and genu and splenium of the corpus callosum-were evaluated on MFC maps in all subjects.

To compute the T2 lesion load, we transferred the dual-echo fast spin-echo (proton attenuation and T2-weighted) image data to a SPARC workstation (Sun Microsystems, Mountain View, Calif). We performed segmentation of the T2 lesions using the 3D VIEWNIX software system with the concept of "fuzzy connectedness." 25,26 The MS lesions, identified as 3D fuzzy objects, and the voxels belonging to the lesions were created as $3 \mathrm{D}$ volume images, which provided the total number and volume of the lesions. This method has been validated previously, and the reproducibility was shown to be $>99 \%$ for $\mathrm{T} 2$ lesion segmentation.

\section{Statistical Analysis}

We used SAS version 9.0 (SAS Institute, Cary, NC) software for all statistical computations. We used least squares (LS) regression to compare the patients and control subjects with respect to the mean MFC generated from both sides of the deep gray matter and white 


\begin{tabular}{lccc}
\hline $\begin{array}{l}\text { Mean and SDs of MFC measure in different brain regions between } \\
\text { patients with relapsing-remitting MS and control subjects* }\end{array}$ \\
\hline & Patients & Controls & $\begin{array}{c}P \\
\text { Value }\end{array}$ \\
Region & $867.7 \pm 177.0$ & $699.7 \pm 145.0$ & .007 \\
\hline Globus pallidus & $540.0 \pm 144.6$ & $387.0 \pm 106.4$ & .002 \\
Putamen & $247.2 \pm 69.0$ & $189.3 \pm 71.6$ & .03 \\
Thalamus & $94.7 \pm 27.4$ & $91.5 \pm 41.9$ & .81 \\
Frontal white matter & $190.9 \pm 44.4$ & $174.1 \pm 45.5$ & .67 \\
Genu of corpus callosum & $108.3 \pm 34.8$ & $103.0 \pm 34.4$ & .31 \\
Splenium of corpus callosum
\end{tabular}

${ }^{*}$ Least squares regression was used to compare patients and controls with respect to the mean MFC generated from both sides of the deep gray matter and white matter regions while $P$ values were adjusted for age and sex.

matter regions while adjusting for age and sex. A separate regression analysis was conducted for each brain region. In each case, the mean value of MFC, averaged over the left and right sides of the given brain region, constituted the dependent variable. The regression model included sex and group (MS group versus control group) as a classification factor and age of the subjects at the time of image acquisition as a numeric covariate. The error variance was allowed to be different for patients and controls to account for the likely association between the mean and variance of MFC in the progression across the groups. We characterized the association of MFC in each brain region with the number and volume of lesions using Pearson correlation coefficients and assessed for statistical significance with LS regression. The regression analysis followed the outline described above, except that only the patients contributed data to the analysis. The assessment of lesion load (either lesion count or volume, in separate analyses) was added to the model as a numeric factor. We used type $3 P$ values to assess the significance of differences between patients and controls and the association between MFC and lesion count, adjusting for age and sex. Results were declared statistically significant at the 2 -sided 5\% comparison-wise significance level.

\section{Results}

The mean and SD of the MFC values of the deep gray matter (globus pallidus, putamen, and thalamus) and white matter regions (frontal white matter and splenium and genu of the corpus callosum) in the patients and controls are listed in the Table. There were significantly higher MFC values in the patients than in the controls in all 3 deep gray matter regions, indicating abnormal accumulation of iron in these regions. Across the deep gray matter regions in both control and patient groups, the MFC value is highest in the globus pallidus and lowest in the thalamus. Compared with the healthy control subjects, the patients had an average increase of $24 \%$ in the globus pallidus, $39.5 \%$ in the putamen, and $30.6 \%$ in the thalamus. The white matter regions generally had lower values of MFC compared with the MFC in the deep gray matter regions. We found increased MFC in some, but not all, lesions. Figure 1 shows representative images from a patient and a control subject that demonstrate an increased area of MFC in the deep gray matter region on scaled MFC color maps, which is not obvious on conventional T2-weighted images. However, there was no statistical difference of MFC in any of the white matter regions between the patients and the control subjects $(P>.3)$.

We also evaluated the relationship between the patients' individual mean MFC in each of the deep gray matter regions and lesion load, which included the total number and volume of lesions. The correlation coefficients associated with signifi- cant findings from the regression analysis of the relationship between MFC and the total number of lesions in the patients were found in the thalamus $(r=0.61, P=.01)$ and globus pallidus ( $r=0.52, P=.02$ ). Figure 2 shows a graph that suggests a positive correlation between the mean MFC in the thalamus and number of lesions: the higher the number of lesions in the distal white matter regions, the greater the accumulation of iron in the deep gray matter. However, the correlation between MFC in the deep gray matter and volume of lesions was not significant. There was no obvious relationship that could be established between MFC and the white matter regions with lesion load.

Compared with the healthy controls, the patients performed significantly worse on the SDMT neuropsychologic tests $(P=.01)$. With respect to MFC, on the basis of the Spearman correlation coefficient, we found a moderate but significant correlation between the MFC value in the thalamus and the CVLT test $(r=-0.42, P=.04)$ and the RCFT test ( $r=$ $-0.50, P=.03)$. The MFC value in the putamen correlated with the DSB test $(r=0.45, P=.03)$. We performed both the Pearson and Spearman correlation coefficients to assess the relationship between MFC in each brain region and duration of disease. No statistically significant correlation was found, except a trend between MFC in the globus pallidus and duration of disease on the Spearman analysis $(r=0.42, P=.09)$.

\section{Discussion}

MFC methodology has been recently described and validated by Jensen et al. ${ }^{22}$ MFC imaging requires ASE images to be obtained for a fixed acquisition time but with a range of shifts for the $180^{\circ}$ refocusing pulse. It is developed based on a direct relationship to the MFIs generated by spatial variations in magnetic susceptibility. Information about these types of microscopic magnetic tissue structures is therefore encoded in the MR signal intensity to generate MFC map. The MFIs from, for example, iron rich cells in the basal ganglia are generally microscopic MFIs with length scales usually less than $100 \mu \mathrm{m}$. Compared with the conventional measure of transverse relaxation rates, $\mathrm{R} 2$ and $\mathrm{R} 2{ }^{\star},{ }^{27,28} \mathrm{MFC}$ is a more specific metric of microscopic MFIs. In particular, both R2 and R2* depend strongly on molecular dipole-dipole interactions, which have no effect on the MFC. The MFC can also be affected by macroscopic field gradients, but this is likely to give a small contribution except in brain regions near large veins or air/tissue interfaces. ${ }^{24}$ Of further note is that over the full range of brain iron concentrations, the dynamic range of $\mathrm{R} 2$ is limited from approximately 13 to 24 seconds $^{-1}$ whereas MFC ranges from approximately 100 to 1000 seconds $^{-2}$. This suggests that factors other than iron have a larger influence on the measurement of R2 than on the MFC. Because the MFC has a more direct relationship to MFIs than do the conventional quantities of R2 and R2*, it allows for a clearer physical interpretation. The previously shown high correlation ${ }^{24}$ between the MFC values and nonheme iron indicates that MFC imaging may be a better quantitative method for measuring the iron concentration of brain tissue than other conventional techniques.

Deposition of iron has been observed previously on con- 

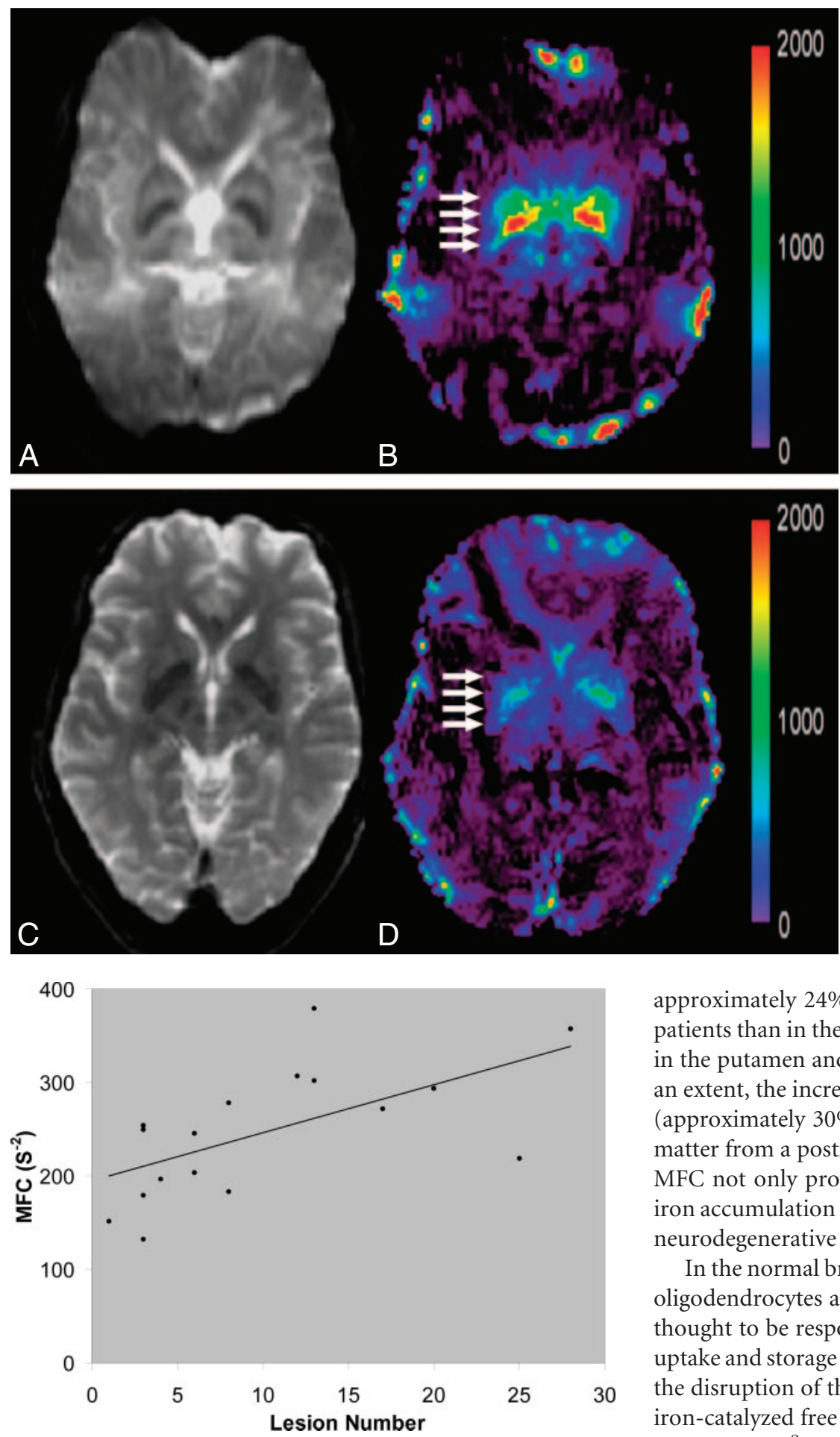

Fig 2. Graph showing significant correlation of MFC in the thalamus and number of lesions in patients with relapsing-remitting MS. This trend indicates the role of white matter lesions (with axonal transection) in the accumulation of iron in deep gray matter by interruption of iron transport from the deep gray matter nuclei to the cortical regions.

ventional MR images in MS. Drayer et al, ${ }^{16}$ in 1987 , observed hypointensity on conventional MR imaging in the deep gray matter regions in MS, in which the decreased signal intensities were presumed to be from iron content. However, their findings were contradictory to those by Grimaud et al, ${ }^{29}$ who reported that only a mild degree of low signal intensity in the thalamus and low signal intensity in the basal ganglia are rarely
Fig 1. T2-weighted images (left) and MFC color maps (right) in a 29-year-old patient with MS (top row) and a 32-year-old control subject (bottom row). Note the clearly increased MFC in the deep gray matter regions on MFC maps (arrows) in the patient $(B)$ relative to the contro subject $(D)$, though such a difference is not remarkable on conventional T2-weighted images $(A, C)$.

seen. More recently, Bakshi et al, ${ }^{15}$ in 2001, have confirmed abnormal hypointensity on T2-weighted imaging not only in the deep gray matter but also in the cortical regions. These inconsistent findings are all from qualitative evaluation by subjective visual impression or rating and highlight the need for a more objective quantitative method for iron measurement. Although T2 signal intensities were measured in the deep gray matter in several studies, ${ }^{19,30}$ $\mathrm{T} 2$ intensity is not specific, and T2weighted sequences (eg, fast spin-echo imaging) are not sensitive to iron components. However, an excellent correlation $(r=0.975)$ between in vivo MFC measurements and brain iron concentration estimates derived from postmortem studies was found in our previous study. ${ }^{24}$ Our data also showed that the increased iron deposition could be clearly demonstrated on the quantitative MFC maps but not on conventional T2-weighted imaging (Fig 1). In this quantitative study, we found the MFC, a more specific measure of iron, increased approximately $24 \%$ to $39.5 \%$ in the deep gray matter in the patients than in the control subjects and was more prominent in the putamen and thalamus than in the globus pallidus. To an extent, the increased MFC was similar to the neuronal loss (approximately $30 \%$ to $35 \%$ reduction) in the thalamic gray matter from a postmortem study. ${ }^{3}$ The quantitative ability of MFC not only provides accurate information of the level of iron accumulation but also offers a tool for the evaluation of a neurodegenerative pathologic process.

In the normal brain, higher levels of iron are found in both oligodendrocytes and the deep gray matter nuclei, which are thought to be responsible for myelinogenesis ${ }^{31}$ and excessive uptake and storage of iron, ${ }^{32}$ respectively. It is also known that the disruption of the normal iron homeostatic status leads to iron-catalyzed free radicals that result in oxidative damage in these regions. ${ }^{9}$ T2 shortening in the deep and cortical gray matter was found to be related to brain atrophy, ${ }^{7,15}$ which indicated destructive effects by abnormal deposition of iron in MS. The precise mechanism of the excessive accumulation of iron in the deep gray matter in MS remains unclear. However, one possible mechanism is related to the disruption of normal iron transport in the neurons of the deep gray matter that have high uptake of iron. ${ }^{33}$ Neurons, especially ferruginated neurons such as in the globus pallidus, not only have extensive expression of the transferrin receptor but also express the ferrous iron transporter, which allows for the detachment of iron within recycling endosomes and the transport of iron to the 
distal cytosol via the axons. ${ }^{32}$ In a histopathologic study, ${ }^{34}$ myelinated white matter and axons near the MS lesions contained numerous iron-laden ovoid bodies that stained positively for iron, which indicated that the possible iron source was the interruption of iron transport and axonal transection by the lesions. Our finding of a positive correlation between MFC and the number of lesions further supports this theory and indicates that lesions in the white matter can disrupt the axonal outflow of iron, which leads to accumulation of iron in the deep gray matter.

We can interpret the positive correlation of MFC with the number of lesions, rather than the lesion volume, indicating that the number of multifocal MS lesions may be more representative of the extent or span of the disease than volume in these patients, though this also could be the result of the limited number of patients in our study. Our results that iron accumulates in the deep gray matter are supported by previous studies that were based on the analysis of T2 hypointensity in MS. ${ }^{15,35}$ The positive correlation between the total number of lesions and MFC in the thalamus (Fig 2) suggests a connecting relationship between the lesions in the distal white matter and iron deposition in the thalamus. The thalamus is a large, central relay station for processing information throughout the brain, with clusters of discrete nuclei and multiple functional pathways connecting associative brain regions. Therefore, the level of iron can be significantly increased (30.6\%) in the thalamus because of the disruption of iron outflow by the lesions on the pathways, despite the normal level of iron in the thalamus being lower compared with other deep gray matter nuclei. Our findings, which included only a trend correlation between duration of disease and MFC in the regions of the deep gray matter, suggested that accumulation of iron can occur at an early stage of MS when enough lesions are present. The lesions then block the output connection of iron from the deep gray matter regions.

However, we have not found significantly increased MFC in normal-appearing white matter regions, including the frontal white matter and corpus callosum, in patients. This observation suggests that the mechanisms of white matter and deep gray matter injury in MS may be different. In an earlier histopathologic study of MS, Craelius et $\mathrm{al}^{34}$ demonstrated that nonheme iron was present as hemosiderin surrounding demyelinated plaques on Perls acid ferrocyanide stain. The role of iron in the pathogenesis of MS has also been implicated in EAE animal model studies, in which there was increased histochemical staining of iron within macrophages and also granular staining in extracellular sites in the active and recovery stages. ${ }^{11,14}$ Excess concentration of iron may also contribute to $\mathrm{CD} 4+\mathrm{T}$-cell development in animal studies of $\mathrm{EAE}^{36}$ that is involved in the autoimmune attack of myelin in MS. We did observe increased MFC in some, but not all, lesions on MFC maps, which probably reflects that the degree of disruption of iron transportation is varied and more noticeable in lesions with severe axonal transection.

In the deep gray matter, decreased NAA, ${ }^{5}$ decreased volume, ${ }^{6}$ and decreased attenuation of neuronal cells ${ }^{3}$ have been reported in previous studies of MS, though there were fewer lesions present in these regions. The underlying pathophysiologic process of this rather typical neurodegeneration is still not well known. However, evidence suggests that the increased accumulation of iron and oxidative stress in the deep gray matter may be responsible for these neurodegenerative changes in many disease states. ${ }^{37}$ Our findings of moderate correlations between iron levels measured with MFC and neuropsychologic data indicate that the excess iron-induced neuronal cell injury is, at least, one of the most important factors for the pathophysiologic process of neuropsychologic deficits. Recently, particular attention has been given to the therapeutic strategy of pharmacologic intervention that targets iron and iron management for the treatment of EAE and MS. Knowing each step of the neurotoxic cascade of secondary tissue injury to iron overload, which generates free radicals and lipid peroxidation, can create a potential target of therapeutic intervention. For example, Desferal, an iron chelator administered in an animal EAE study, showed beneficial results by reducing both the duration and severity of disease. ${ }^{38,39}$

\section{Conclusion}

Our study demonstrated that the severity of iron deposition in the deep gray matter can be measured in MS with the use of quantitative MFC imaging. MFC is a more specific measure of the disruption of normal brain iron homeostasis and of follow-up studies. Investigation of such excessive accumulation of iron in the deep gray matter, with its toxic effects of oxidative stress injury, may help us better understand the neurodegenerative pathologic process and neuropsychologic dysfunction of the disease.

\section{Acknowledgments}

The authors thank Hina Jaggi for the recruitment of subjects and Kamila Szulc for technical assistance.

\section{References}

1. Minagar A. Gray matter involvement in multiple sclerosis: a new window into pathogenesis. J Neuroimaging 2003;13:291-92

2. Filippi M. Multiple sclerosis: a white matter disease with associated gray matter damage. J Neurol Sci 2001;185:3-4

3. Cifelli A, Arridge M, Jezzard $\mathrm{P}$, et al. Thalamic neurodegeneration in multiple sclerosis. Ann Neurol 2002;52:650-53

4. Wylezinska M, Cifelli A, Jezzard P, et al. Thalamic neurodegeneration in relapsing-remitting multiple sclerosis. Neurology 2003;60:1949-54

5. Inglese M, Liu S, Babb JS, et al. Three-dimensional proton spectroscopy of deep gray matter nuclei in relapsing-remitting MS. Neurology 2004;631:170-72

6. Carone DA, Benedict RH, Dwyer MG, et al. Semi-automatic brain region extraction (SABRE) reveals superior cortical and deep gray matter atrophy in MS. Neuroimage 2006;29:505-14

7. Bermel RA, Puli SR, Rudick RA, et al. Prediction of longitudinal brain atrophy in multiple sclerosis by gray matter magnetic resonance imaging $\mathrm{T} 2$ hypointensity. Arch Neurol 2005;62:1371-76

8. Bartzokis G, Tishler TA, Shin IS, et al. Brain ferritin iron as a risk factor for age at onset in neurodegenerative diseases. Ann N Y Acad Sci 2004;1012:224-36

9. Campbell A, Smith MA, Sayre LM, et al. Mechanisms by which metals promote events connected to neurodegenerative diseases. Brain Res Bull 2001;55:125-32

10. Levine SM, Chakrabarty A. The role of iron in the pathogenesis of experimental allergic encephalomyelitis and multiple sclerosis. Ann N Y Acad Sci 2004;1012:252-66

11. Xu S, Jordan EK, Brocke S, et al. Study of relapsing remitting experimental allergic encephalomyelitis SJL mouse model using MION-46L enhanced in vivo MRI: early histopathological correlation. J Neurosci Res 1998;52:549-58

12. Mehindate K, Sahlas DJ, Frankel D, et al. Proinflammatory cytokines promote glial heme oxygenase-1 expression and mitochondrial iron deposition: implications for multiple sclerosis. J Neurochem 2001;77:1386-95

13. Ruuls SR, Bauer J, Sontrop K, et al. Reactive oxygen species are involved in the pathogenesis of experimental allergic encephalomyelitis in Lewis rats. $\mathrm{J} \mathrm{Neu}$ roimmunol 1995;56:207-17

14. Forge JK, Pedchenko TV, LeVine SM. Iron deposits in the central nervous 
system of SJL mice with experimental allergic encephalomyelitis. Life Sci 1998;63:2271-84

15. Bakshi R, Dmochowski J, Shaikh ZA, et al. Gray matter T2 hypointensity is related to plaques and atrophy in the brains of multiple sclerosis patients. J Neurol Sci 2001;185:19-26

16. Drayer BP, Burger P, Hurwitz B, et al. Magnetic resonance imaging in multiple sclerosis: decreased signal in thalamus and putamen. Ann Neurol 1987;22:546-50

17. Amato MP, Ponziani G, Siracusa G, et al. Cognitive dysfunction in early-onset multiple sclerosis: a reappraisal after 10 years. Arch Neurol 2001;58:1602-06

18. Ge Y, Udupa JK, Nyul LG, et al. Numerical tissue characterization in MS via standardization of the MR image intensity scale. J Magn Reson Imaging 2000;12:715-21

19. Bakshi R, Benedict RH, Bermel RA, et al. T2 hypointensity in the deep gray matter of patients with multiple sclerosis: a quantitative magnetic resonance imaging study. Arch Neurol 2002;59:62-68

20. Jensen JH, Johnson G, Chandra R, et al. MRI measurement of magnetic field correlation in a cell suspension. Proc Intl Soc Mag Reson Med Berkeley, Calif: International Society for Magnetic Resonance in Medicine. Toronto, Canada; 2003;11:1120

21. Helpern JA, Jensen J, Lee SP, et al. Quantitative MRI assessment of Alzheimer's disease. J Mol Neurosci 2004;24:45-48

22. Jensen JH, Chandra R, Ramani A, et al. Magnetic field correlation imaging. Magn Reson Med 2006;55:1350-61

23. Jensen JH, Chandra R. Method for measuring the magnetic field correlation function for water protons in biological tissues. Proc Intl Soc Mag Reson Med Berkeley, Calif: International Society for Magnetic Resonance in Medicine. Honolulu, Hawaii; 2002;10:2297

24. Ramani A, Jensen $\mathrm{JH}$, Krain $\mathrm{AL}$, et al. In vivo detection of age-related brain iron differences with MFC. Proc Intl Soc Mag Reson Med Berkeley, Calif: International Society for Magnetic Resonance in Medicine. Seattle, Wash; 2006;14:1559

25. Udupa JK, Odhner D, Samarasekera S, et al. 3D VIEWNIX: an open, transportable, multidimensional, multimodality, multiparametric imaging software system. Proc Intl Soc Optical Eng 1994;2164:58-73

26. Udupa JK, Wei L, Samarasekera S, et al. Multiple sclerosis lesion quantifi- cation using fuzzy-connectedness principles. IEEE Trans Med Imaging 1997; 16:598-609

27. Miszkiel KA, Paley MN, Wilkinson ID, et al. The measurement of R2, R2 ${ }^{\star}$ and R2' in HIV-infected patients using the prime sequence as a measure of brain iron deposition. Magn Reson Imaging 1997;15:1113-19

28. Clark PR, Chua-anusorn W, St Pierre TG. Bi-exponential proton transverse relaxation rate (R2) image analysis using RF field intensity-weighted spin density projection: potential for R2 measurement of iron-loaded liver. Magn Reson Imaging 2003;21:519-30

29. Grimaud J, Millar J, Thorpe JW, et al. Signal intensity on MRI of basal ganglia in multiple sclerosis. J Neurol Neurosurg Psychiatry 1995;59:306-08

30. Tjoa CW, Benedict RH, Weinstock-Guttman B, et al. MRI T2 hypointensity of the dentate nucleus is related to ambulatory impairment in multiple sclerosis. J Neurol Sci 2005;234:17-24

31. LeVine SM, Macklin WB. Iron-enriched oligodendrocytes: a reexamination of their spatial distribution. J Neurosci Res 1990;26:508-12

32. Moos T, Morgan EH. The metabolism of neuronal iron and its pathogenic role in neurological disease: review. Ann N Y Acad Sci 2004;1012:14-26

33. Dietrich RB, Bradley WG Jr. Iron accumulation in the basal ganglia following severe ischemic-anoxic insults in children. Radiology 1988;168:203-06

34. Craelius W, Migdal MW, Luessenhop CP, et al. Iron deposits surrounding multiple sclerosis plaques. Arch Pathol Lab Med 1982;106:397-99

35. Drayer B, Burger P, Hurwitz B, et al. Reduced signal intensity on MR images of thalamus and putamen in multiple sclerosis: increased iron content? AJR Am J Roentgenol 1987;149:357-63

36. Grant SM, Wiesinger JA, Beard JL, et al. Iron-deficient mice fail to develop autoimmune encephalomyelitis. J Nutr 2003;133:2635-38

37. Thompson KJ, Shoham S, Connor JR. Iron and neurodegenerative disorders. Brain Res Bull 2001;55:155-64

38. Bowern N, Ramshaw IA, Clark IA, et al. Inhibition of autoimmune neuropathological process by treatment with an iron-chelating agent. J Exp Med 1984;160:1532-43

39. Pedchenko TV, LeVine SM. Desferrioxamine suppresses experimental allergic encephalomyelitis induced by MBP in SJL mice. J Neuroimmunol 1998;84: $188-97$ 